\title{
Technology Selection in the Presence of Imprecise Data, Weight Restrictions, and Nondiscretionary Factors
}

\author{
Reza Farzipoor Saen \\ Iran, P. O. Box: 31485-313 \\ Tel: 0098 (261) 4418144-6 \\ Fax: 0098 (261) 4418156 \\ Email: farzipour@yahoo.com
}

Department of Industrial Management, Faculty of Management and Accounting, Islamic Azad University-Karaj Branch, Karaj,

\begin{abstract}
Technology selection is an important part of management of technology. Traditionally, technology selection models are based on cardinal data with less emphasis on ordinal data. However, with respect to technology selection complexity, emphasis has shifted to the simultaneous consideration of cardinal and ordinal data in technology selection process. The application of Data Envelopment Analysis (DEA) for technology selection problems is based on total flexibility of the weights. However, the problem of allowing total flexibility of the weights is that the values of the weights obtained by solving the unrestricted DEA program are often in contradiction to prior views or additional available information. On the other hand, current models of technology selection problems assume complete discretionary of decision making criteria and do not assume technology selection in the conditions that some factors are nondiscretionary. To select the best technologies in the presence of cardinal data, ordinal data, nondiscretionary factors, and weight restrictions the objective of this paper is to propose a new pair of Assurance Region-Nondiscretionary Factors-Imprecise Data Envelopment Analysis (AR-NF-IDEA) models. A numerical example demonstrates the application of the proposed method.
\end{abstract}

Keywords: Technology selection, Imprecise data envelopment analysis, Nondiscretionary factors, Assurance region

\section{Introduction}

Selecting the right technology is always a difficult task for decision makers. Technologies have varied strengths and weaknesses which require careful assessment by the purchasers. Technology selection models help decision maker choose between evolving technologies. The reason for a special focus on technology selection is due to the complexity of their evaluation which includes strategic and operational characteristics. Tools that consider a wide range of dimensions have been developed for evaluating these many characteristics, which include cost, quality, flexibility, time, etc.

One of the uses of Data Envelopment Analysis (DEA) is technology selection. In original DEA formulations the assessed Decision Making Units (DMUs) can freely choose the weights or values to be 
assigned to each input and output in a way that maximizes its efficiency, subject to this system of weights being feasible for all other DMUs. This freedom of choice shows the DMU in the best possible light, and is equivalent to assuming that no input or output is more important than any other. The free imputation of input-output values can be seen as an advantage, especially as far as the identification if inefficiency is concerned. If a DMU (technology) is free to choose its own value system and some other technology uses this same value system to show that the first technology is not efficient, then a stronger statement is being made. The advantages of full flexibility in identifying inefficiency can be seen as disadvantages in the identification of efficiency. An efficient technology may become so by assigning a zero weight to the inputs and/or outputs on which its performance is worst. This might not be acceptable by Decision Makers (DMs) as well as by the analyst, who after spending time in a careful selection of inputs and outputs sees some of them being completely neglected by technology. DMs may have in technology selection problems value judgments that can be formalized a priori, and therefore should be taken into account in technology selection. These value judgments can reflect known information about how the factors used by the technologies behave, and/or "accepted" beliefs or preferences on the relative worth of inputs, outputs or even technologies. For example, suppose we want to buy a dot-matrix printer and we may, given the price, make trade-offs amongst the speed, print quality, and input buffer (memory) which are some of the most important features that distinguish 24-pin dot-matrix printers. We may not consider the printer memory feature to be very vital, because dot-matrix printers only use memory as a buffer space to download fonts. Thus, more consideration is given to speed and print quality. Perhaps, the printer is simply used to print long program codes or data-base listings, so that speed outweighs print quality. Therefore, in technology selection, incorporation of value judgment is very important and ignoring this aspect may lead to biased efficiency results. To avoid the problem of free (and often undesirable) specialization, input and output weights should be constrained in DEA.

Nevertheless, technology selection models are based on cardinal data with less emphasis on ordinal data. However, with respect to technology selection complexities, emphasis has shifted to the simultaneous consideration of cardinal and ordinal data in technology selection process.

On the other hand, discretionary models for evaluating the efficiency of technologies assume that all criteria are discretionary, i.e., controlled by the management of each technology supplier and varied at its discretion. Thus, failure of a technology to produce maximal output levels with minimal input consumption results in a decreased efficiency score. In any realistic situation, however, there may exist exogenously fixed or nondiscretionary criteria that are beyond the control of a management. For example, if the technology under analysis has limited number of suppliers (oligopolistic environment), then cost of technology will be a nondiscretionary factor, i.e., cost reduction will not be acceptable from the technology supplier's perspective.

This paper depicts the technology selection process through an Imprecise Data Envelopment Analysis (IDEA) model, while allowing for the incorporation of nondiscretionary factors and weight restrictions. 
The objective of this paper is to propose a new pair of Assurance Region-Nondiscretionary FactorsImprecise Data Envelopment Analysis (AR-NF-IDEA) models for selecting the best technologies in the presence of weight restrictions, nondiscretionary factors and imprecise data.

This paper proceeds as follows. In Section 2, literature review is presented. Section 3 discusses the proposed method for technology selection. Numerical example and concluding remarks are discussed in Sections 4 and 5, respectively.

\section{Literature review}

Some mathematical programming approaches have been used for technology selection in the past. Rai et al. [1] addressed application of a fuzzy Goal Programming (GP) concept to model the problem of machine-tool selection and operation allocation with explicit considerations given to objectives of minimizing the total cost of machining operation, material handling and setup. A genetic algorithm based approach is adopted to optimize the fuzzy GP. Chan et al. [2] presented a fuzzy GP approach to model the machine tool selection and operation allocation problem of Flexible Manufacturing Systems (FMSs). However, one of the GP problems arises from a specific technical requirement. After the DM specify the goals for each selected criterion, they must decide on a preemptive priority order of these goals, i.e., determining in which order the goals will be attained. Frequently such a priori input might not produce an acceptable solution and the priority structure may be altered to resolve the problem once more. In this fashion, it may be possible to generate a solution iteratively that finally satisfies the DM. Unfortunately, the number of potential priority reorderings may be very large. A technology selection problem with five factors has up to 120 priority reorderings. Going through such a laborious process would be costly and inefficient.

Jaganathan et al. [3] proposed an integrated fuzzy Analytic Hierarchy Process (AHP) based approach to facilitate the selection and evaluation of new manufacturing technologies in the presence of intangible attributes and uncertainty. However, AHP has two main weaknesses. First subjectivity of AHP is a weakness. Second AHP could not include interrelationship within the criteria in the model.

Because of the complexity of the decision making process involved in technology selection, all the aforementioned literature relied on some form of procedures that assigns weights to various performance measures. The primary problem associated with arbitrary weights is that they are subjective, and it is often a difficult task for the DM to accurately assign numbers to preferences. It is a daunting task for the DM to assess weighting information as the number of performance criteria increased. Therefore, a more robust mathematical technique that does not demand too much and too precise information, i.e., ordinal preferences instead of cardinal weights, from the DM can strengthen the technology evaluation process. To this end, Khouja [4] proposed a decision model for technology selection problems using a two-phase procedure. In phase 1, DEA is used to identify technologies that provide the best combinations of vendor specifications on the performance parameters of the technology. In phase 2, a MADM model is used to 
select a technology from those identified in phase 1. Khouja [4] used MADM, to select a robot from the efficient robots. Baker and Talluri [5] proposed an alternate methodology for technology selection using DEA. They addressed some of the shortcomings in the methodology suggested by Khouja [4] and presented a more robust analysis based on cross-efficiencies in DEA. However, a factor which possibly reduces the usefulness of cross-efficiency is that the weights that maximize DMU simple efficiency may be not unique. The same result can be achieved via different solutions. Because the choice of the weights influences the cross-efficiency measurements for the other DMUs, its casualness may imply distorted judgments. Ramanathan [6] introduced the use of DEA for synthesizing the diverse characteristics of energy supply technologies into a single objective efficiency score. It has been found that, of the eight technologies considered, nuclear and solar photovoltaic technologies were rated to be the most relatively efficient. To select the best technologies in the existence of both cardinal and ordinal data, Farzipoor Saen [7] proposed an innovative approach, which is based on IDEA. Talluri et al. [8] proposed a framework, which is based on the combined application of DEA and nonparametric statistical procedures, for the selection of FMSs. The strengths of this methodology are that it incorporates variability measures in the performance of alternative systems, provides DM with effective alternative choices by identifying homogeneous groups of systems, and presents graphic aids for better interpretation of results. Seiford and Zhu [9] extended the context-dependent DEA by incorporating value judgment into the attractiveness and progress measures. The method was applied to measuring the attractiveness of 32 computer printers. They showed that the attractive measure helps (i) customers to select the best option, and (ii) printer manufacturers to identify the potential competitors. To select the best advanced manufacturing technologies, Karsak and Ahiska [10] introduced a multi-criteria decision methodology that can integrate multiple outputs such as various technical characteristics and qualitative factors with a single input such as cost. Their model is derived from the cross-efficiency analysis, which is one of the branches of DEA model. Sarkis and Talluri [11] introduced an application of DEA that considers both cardinal and ordinal data, for the evaluation of alternative FMS. The DEA models proposed integrate both qualitative and quantitative data. The initial DEA model is based on the works of Cook et al. [12]. To improve the discriminatory power of DEA in the existence of both cardinal and ordinal factors, an additional DEA model relying on pairwise comparisons of FMS was proposed. The results of the pairwise comparison model are aggregated through cross-efficiency measures. Talluri and Yoon [13] introduced advanced manufacturing technology selection process. They proposed a combination of a cone-ratio DEA model and a new methodological extension in DEA, while allowing for the incorporation of preferences of decision makers. Shang and Sueyoshi [14] utilized a combination of AHP and DEA for selection of FMS. Their model used AHP to restrict factor weights in DEA. However, they could not solve the difficulty with multiple optimum solutions. Braglia and Petroni [15] proposed the use of DEA for selection of industrial robots. Their methodology is based on a sequential dual use of DEA with restricted weights. To restrict the weights, they suggested a rule of thumb. 
However, all the aforementioned references are based on complete discretionary of decision making criteria (factors) and do not consider technology selection in the presence of weight restrictions, imprecise data and nondiscretionary factors. Although [16], and [17] proposed to employ DEA for selecting the best technologies in the existence of nondiscretionary factors, but they did not introduce a model which selects the technologies in the presence of weight restrictions, imprecise data and nondiscretionary factors. In other words, they did not consider weight restrictions and imprecise data. To the best of author's knowledge, there is not any reference that discusses technology selection in the presence of weight restrictions, imprecise data and nondiscretionary factors. The approach presented in this paper has some distinctive features.

- The proposed model considers cardinal and ordinal data for technology selection.

- The proposed model considers nondiscretionary factors for technology selection.

- The proposed model considers weight restrictions for technology selection.

- Technology selection is a straightforward process carried out by the proposed model.

- Imprecise data, weight restrictions, and nondiscretionary factors are considered simultaneously.

- The proposed model deals with imprecise data in a direct manner.

- The results of this paper are not only valuable to buyers, but to technology suppliers as well. The buyer will be given the advice to avoid certain technologies as being inefficient for one or more reasons. The technology supplier will know if the competitor's presumptive technology is superior to its own and in that case what substantial improvements are required in order to turn its inefficient technology into an efficient one.

- An application of the methodology has been performed on a set of data retrieved from the information of 27 robots.

\section{Proposed method for technology selection}

DEA proposed by Charnes et al. [18] (CCR model) and developed by Banker et al. [19] (BCC model) is an approach for evaluating the efficiencies of DMUs. This evaluation is generally assumed to be based on a set of cardinal (quantitative) output and input factors. In many real world applications (especially technology selection problems), however, it is essential to take into account the existence of ordinal (qualitative) factors when rendering a decision on the performance of a DMU. Very often it is the case that for a factor such as vendor reputation, one can, at most, provide a ranking of the DMUs from best to worst relative to this attribute. The capability of providing a more precise, quantitative measure reflecting such a factor is generally beyond the realm of reality. In some situations, such factors can be legitimately quantified, but very often; such quantification may be superficially forced as a modeling convenience. In situations such as that described, the data for certain influence factors (inputs and outputs) might better be represented as rank positions in an ordinal, rather than numerical sense. Refer again to the vendor 
reputation example. In certain circumstances, the information available may permit one to provide a complete rank ordering of the DMUs on such a factor. Therefore, the data may be imprecise.

On the other hand, one serious drawback of DEA applications in technology selection has been the absence of DM judgment, allowing total freedom when allocating weights to input and output data of technology under analysis. This allows technologies to achieve artificially high efficiency scores by indulging in inappropriate input and output weights. The most widespread method for considering judgments in DEA models is, perhaps, the weight restrictions inclusion. Weight restrictions allow for the integration of managerial preferences in terms of relative importance levels of various inputs and outputs. The idea of conditioning the DEA calculations to allow for the presence of additional information arose first in the context of bounds on factor weights in DEA's multiplier side problem. This led to the development of the cone-ratio (Charnes et al. [20]) and Assurance Region (AR) models (Thompson et al. [21]). Both methods constrain the domain of feasible solutions in the space of the virtual multipliers. Weights restrictions may be applied directly to the DEA weights or to the product of these weights with the respective input or output level, referred to as virtual input or virtual output. Restrictions on virtual weights were proposed first by Wong and Beasley [22]. Sarrico and Dyson [23] suggest, in line with Thompson et al. [21], the use of virtual assurance regions, concluding that they can overcome problems of infeasibility as well as interpretation of target and efficiency scores, whilst retaining the benefit of the natural representation of preference structures. In this paper, this concept is used to restrict the flexibility of the technologies (DMUs) in selecting the weights.

To the best of author's knowledge, the only papers that discuss IDEA in the presence of weight restrictions are the works of Cooper et al. [24], Cooper et al. [25], and Cooper et al. [26]. In these papers, the AR-IDEA model was developed to deal not only with imprecise data but also with weight restrictions. However, their method makes the DEA model become very complicated because of great numbers of data transformations and variable alternations. Furthermore, in dealing with the bounded data, their model requires that bounded outputs and inputs at least have one exact (maximum) data. Recently, Wang et al. [27] developed a new pair of interval DEA models for dealing with imprecise data such as interval data, ordinal preference information, fuzzy data and their mixture. Compared with the IDEA model developed by Cooper et al. [24], Cooper et al. [25], and Cooper et al. [26], their interval DEA models are much easier to understand and more convenient to use. Also, compared with the interval DEA models developed by Despotis and Smirlis [28], their interval DEA models utilize a fixed and unified production frontier as a benchmark to measure the efficiencies of all DMUs, which makes their models more rational and more reliable. Moreover, the means they treat ordinal preference information also seems more reasonable than the way Zhu [29] did. However, Wang et al. [27] did not consider weight restrictions and nondiscretionary factors.

In this section, a new pair of AR-NF-IDEA models is proposed that can overcome the shortcomings mentioned above, to consider nondiscretionary factors of the technologies (DMUs) while imprecise data 
and weight restrictions are present. The final efficiency score for each DMU will be characterized by an interval bounded by the best lower bound efficiency and the best upper bound efficiency of each DMU.

Suppose that there are $n$ DMUs to be evaluated. Each DMU consumes $m$ inputs to produce $s$ outputs. In particular, $\mathrm{DMU}_{j}$ consumes amounts $\mathrm{X}_{j}=\left\{x_{i j}\right\}$ of inputs $(i=1, \ldots, m)$ and produces amounts $\mathrm{Y}_{\mathrm{j}}=\left\{y_{r j}\right\}$ of outputs $(r=1, \ldots, s)$. Without loss of generality, it is assumed that all the input and output data $x_{i j}$ and $y_{r j}$ $(i=1, \ldots, m ; r=1, \ldots, s ; j=1, \ldots, n)$ cannot be exactly obtained due to the existence of uncertainty. They are only known to lie within the upper and lower bounds represented by the intervals $\left\lfloor x_{i j}^{L}, x_{i j}^{U}\right\rfloor$ and $\left[y_{r j}^{L}, y_{r j}^{U}\right]$, where $x_{i j}^{L}>0$ and $y_{r j}^{L}>0$.

In order to deal with such an uncertain situation, the following pair of linear programming models have been developed to generate the upper and lower bounds of interval efficiency for each DMU (Wang et al. [27]):

$$
\operatorname{Max} \theta_{j o}^{U}=\sum_{r=1}^{s} u_{r} y_{r j_{o}}^{U}
$$

s.t.

$$
\begin{aligned}
& \sum_{i=1}^{m} v_{i} x_{i j_{o}}^{L}=1, \\
& \sum_{r=1}^{s} u_{r} y_{r j}^{U}-\sum_{i=1}^{m} v_{i} x_{i j}^{L} \leq 0, \quad j=1, \cdots, n, \\
& u_{r}, v_{i} \geq \varepsilon \quad \forall r, i .
\end{aligned}
$$

$$
\operatorname{Max} \theta_{j o}^{L}=\sum_{r=1}^{s} u_{r} y_{r j_{o}}^{L}
$$

s.t.

$$
\begin{aligned}
& \sum_{i=1}^{m} v_{i} x_{i j_{o}}^{U}=1, \\
& \sum_{r=1}^{s} u_{r} y_{r j}^{U}-\sum_{i=1}^{m} v_{i} x_{i j}^{L} \leq 0, \quad j=1, \cdots, n, \\
& u_{r}, v_{i} \geq \varepsilon \quad \forall r, i .
\end{aligned}
$$

where $j_{o}$ is the DMU under evaluation (usually denoted by $\mathrm{DMU}_{o}$ ); $u_{r}$ and $v_{i}$ are the weights assigned to the outputs and inputs; $\theta_{j o}^{U}$ stands for the best possible relative efficiency achieved by $\mathrm{DMU}_{o}$ when all the DMUs are in the state of best production activity, while $\theta_{j o}^{L}$ stands for the lower bound of the best possible relative efficiency of $\mathrm{DMU}_{o}$. They constitute a possible best relative efficiency interval $\left[\theta_{j o}^{L}, \theta_{j o}^{U}\right]$. $\varepsilon$ is the non-Archimedean infinitesimal. 
Note that Model (1) determines the production frontier for all the DMUs and Model (2) uses the production frontier as a benchmark to measure the lower bound efficiency of each DMU. No matter what values the inputs and outputs take for each DMU and no matter what weights the models use, the efficiencies of DMUs are all limited to less than or equal to one. It is also very clear from Models (1) and (2) that the constraint set used to measure the efficiencies of DMUs is completely the same, which is made up of the data set $\left\{\left(x_{i j}^{l}, y_{r j}^{u}\right)(i=1, \ldots, m, r=1, \ldots, s, j=1, \ldots, n)\right\}$. However, it must be pointed out that both the best and the worst production activities of $\mathrm{DMU}_{o}$ are considered in (2), one in the constraint set and the other in objective function.

In order to judge whether a DMU is DEA efficient or not, the following definition is given.

Definition 1. A DMU, $\mathrm{DMU}_{o}$, is said to be DEA efficient if its best possible upper bound efficiency $\theta_{j o}^{U^{*}}=1$; otherwise, it is said to be DEA inefficient if $\theta_{j o}^{U^{*}}<1$.

In (3) the various types of weight restriction that can be applied to multiplier models are shown.

\section{Absolute weight restrictions}

$$
\delta_{i} \leq v_{i} \leq \tau_{i} \quad\left(g_{i}\right) \quad \rho_{r} \leq u_{r} \leq \eta_{r} \quad\left(g_{o}\right)
$$

Assurance region of type I (relative weight restrictions)

$$
\alpha_{i} \leq \frac{v_{i}}{v_{i+1}} \leq \beta_{i} \quad\left(h_{i}\right) \quad \theta_{r} \leq \frac{u_{r}}{u_{r+1}} \leq \zeta_{r}
$$

Assurance regions of type II (input - output weight restrictions)

$$
\gamma_{i} v_{i} \geq u_{r}
$$

The Greek letters $\left(\delta_{i}, \tau_{i}, \rho_{r}, \eta_{r}, \alpha_{i}, \beta_{i}, \theta_{r}, \zeta_{r}, \gamma_{i}\right)$ are user-specified constants to reflect value judgments the DM wishes to incorporate in the assessment. They may relate to the perceived importance or worth of input and output factors. The restrictions $(g)$ and $(h)$ in (3) relate on the left hand side to input weights and on the right hand side to output weights. Constraint (l) links directly input and output weights. Absolute weight restrictions are the most immediate form of placing restrictions on the weights as they simply restrict them to vary within a specific range. Assurance region of type I, link either only input weights $\left(h_{i}\right)$ or only output weights $\left(h_{o}\right)$. The relationship between input and output weights are termed assurance region of type II.

Restrictions on virtual inputs/virtual outputs assume the form in (4), where the proportion of the total virtual output of $\mathrm{DMU}_{j}$ accounted for by output $r$ is restricted to lie in the range $\left[a_{r}, b_{r}\right]$ and the 
proportion of the total virtual input of $\mathrm{DMU}_{j}$ accounted for by input $i$ is restricted to lie in the range $\left[c_{i}, d_{i}\right]$.

$$
\begin{array}{cc}
a_{r} \leq \frac{u_{r} y_{r j}}{\sum_{r=1}^{s} u_{r} y_{r j}} \leq b_{r}, & r=1, \ldots, s \\
c_{i} \leq \frac{v_{i} X_{i j}}{\sum_{i=1}^{m} v_{i} x_{i j}} \leq d_{i}, & i=1, \ldots, m
\end{array}
$$

All proposed virtual weights restrictions can be described by the general set of $w=1, \ldots, t$ weights restrictions, applying to the $\mathrm{DMU}_{o}$ :

$$
\sum_{i=1}^{m} a_{i w} x_{i o} v_{i}+\sum_{r=1}^{s} b_{r w} y_{r o} u_{r} \geq k_{w} \quad \forall w
$$

where $a_{i w}$ is the weight of ith input in wth weight restriction and $b_{r w}$ is the weight of $r$ th output in wth weight restriction.

This expression encapsulates the three different kinds of virtual weights restrictions, of the classification presented below.

\subsection{Absolute virtual weights restrictions}

Absolute virtual weights restrictions involve constraining the virtual of a single factor. This approach is equivalent to using proportional virtual weights restrictions applied to output virtuals in an output oriented model, as $\sum_{r=1}^{s} y_{r o} u_{r}=1$. If applied also to input virtuals in an output oriented model, it will only be equivalent for the DMUs that are efficient (i.e. $\sum_{i=1}^{m} x_{i o} v_{i}=1$ ), and therefore define the frontier. They are of the form:

$$
\begin{array}{ll}
a_{i w} x \geq k, & i=i^{\prime}, \\
a_{i w}=0 & \forall i \neq i^{\prime}, \\
b \quad=0 & \forall r
\end{array}
$$

for restricting the virtual input $i^{\prime}$; and 


$$
\begin{array}{ll}
b_{r w} y_{r o} \geq k_{w}, & r=r^{\prime}, \\
b_{r w}=0 & \forall r \neq r^{\prime}, \\
a_{i w}=0 & \forall i
\end{array}
$$

for restricting the virtual output $r^{\prime}$.

These restrictions are useful when the DM is able to specify particular bounds, or wants to assure that a certain factor attains a threshold value, for instance.

\subsection{Virtual assurance regions of type I}

Assurance regions of type I virtual restrictions, link virtual inputs or outputs to translate an ordering of preference. They are

$$
\begin{array}{ll}
\sum_{i=1}^{m} a_{i w} x_{i o} v_{i} \geq 0, & \\
b_{r w}=0 & \forall r
\end{array}
$$

to link virtual inputs, and

$$
\begin{aligned}
& \sum_{r=1}^{S} b_{r w} y_{r o} u_{r} \geq 0, \\
& a_{i w}=0 \quad \forall i
\end{aligned}
$$

to link virtual outputs.

These restrictions are useful when the DM cannot assign particular bounds to the factors, but is able to decide that a factor is more important than another, twice as important, etc.

\subsection{Virtual assurance regions of type II}

Finally, assurance regions of type II virtual restrictions, link the input-output divide. They can be translated by

$$
\sum_{i=1}^{m} a_{i w} x_{i o} v_{i}+\sum_{r=1}^{S} b_{r w} y_{r o} u_{r} \geq 0
$$

where at least one $a_{i w} \neq 0$ and one $b_{r w} \neq 0$.

These restrictions are useful when there is a known relationship between an input and an output. For instance, it is known that to produce a certain output, one needs to have a certain level of a certain input.

The multipliers formulation, with the virtual weights restrictions applying to all DMUs, is as below 
$\operatorname{Max} \theta_{j o}^{U}=\sum_{r=1}^{S} u_{r} y_{r j_{O}}^{U}$

s.t.

$$
\begin{aligned}
& \sum_{i=1}^{m} v_{i} x_{i j_{O}}^{L}=1, \\
& \sum_{r=1}^{S} u_{r} y_{r j}^{U}-\sum_{i=1}^{m} v_{i} x_{i j}^{L} \leq 0, \quad j=1, \cdots, n, \\
& \sum_{r=1}^{S} b_{r w} y_{r j}^{U} u_{r}+\sum_{i=1}^{m} a_{i w} x_{i j}^{L} v_{i} \geq k_{w} \quad \forall w, j \\
& u_{r}, v_{i} \geq \varepsilon \quad \forall r, i .
\end{aligned}
$$

$\operatorname{Max} \theta_{j o}^{L}=\sum_{r=1}^{S} u_{r} y_{r j_{O}}^{L}$

s.t.

$$
\begin{aligned}
& \sum_{i=1}^{m} v_{i} x_{i j_{O}}^{U}=1, \\
& \sum_{r=1}^{S} u_{r} y_{r j}^{U}-\sum_{i=1}^{m} v_{i} x_{i j}^{L} \leq 0, \quad j=1, \cdots, n, \\
& \sum_{r=1}^{S} b_{r w} y_{r j}^{U} u_{r}+\sum_{i=1}^{m} a_{i w} x_{i j}^{L} v_{i} \geq k_{w} \quad \forall w, j \\
& u_{r}, v_{i} \geq \varepsilon \quad \forall r, i .
\end{aligned}
$$

The envelopment formulation (dual problem) of Models (11) and (12) becomes 
$\operatorname{Minc}_{j o}^{U}=\theta_{O}-\sum_{j=1}^{n} \sum_{w=1}^{t} k_{w} \rho_{w j}-\varepsilon \sum_{i=1}^{m} s_{i}^{-}-\varepsilon \sum_{r=1}^{S} s_{r}^{+}$,

s.t.

$$
\begin{array}{lc}
\sum_{j=1}^{n} y_{r j}^{U} \lambda_{j}-\sum_{j=1}^{n} \sum_{w=1}^{t} b_{r w} y_{r j}^{U} \rho_{w j}-s_{r}^{+}=y_{r j o}^{U} & r=1, \cdots, s, \\
x_{i j o}^{L} \theta_{o}-\sum_{j=1}^{n} x_{i j}^{L} \lambda_{j}-\sum_{j=1}^{n} \sum_{w=1}^{t} a_{i w} x_{i j}^{L} \rho_{w j}-s_{i}^{-}=0 & \text { free, } \\
\theta_{o} & \forall w, j, \\
\rho_{w j} \geq 0 & j=1, \cdots, n, \\
\lambda_{j} \geq 0 & i=1, \cdots, m, \\
s_{i}^{-} \geq 0 & r=1, \cdots, s .
\end{array}
$$

$\operatorname{Minc}_{j o}^{L}=\theta_{o}-\sum_{j=1}^{n} \sum_{w=1}^{t} k_{w} \rho_{w j}-\varepsilon \sum_{i=1}^{m} s_{i}^{-}-\varepsilon \sum_{r=1}^{S} s_{r}^{+}$,

s.t.

$$
\begin{array}{lc}
\sum_{j=1}^{n} y_{r j}^{U} \lambda_{j}-\sum_{j=1}^{n} \sum_{w=1}^{t} b_{r w} y_{r j}^{U} \rho_{w j}-s_{r}^{+}=y_{r j o}^{L} & r=1, \cdots, s, \\
x_{i j o}^{U} \theta_{O}-\sum_{j=1}^{n} x_{i j}^{L} \lambda_{j}-\sum_{j=1}^{n} \sum_{w=1}^{t} a_{i w} x_{i j}^{L} \rho_{w j}-s_{i}^{-}=0 & i=1, \cdots, m, \\
\theta_{O} & \text { free, } \\
\rho_{w j} \geq 0 & \forall w, j, \\
\lambda_{j} \geq 0 & j=1, \cdots, n, \\
s_{i}^{-} \geq 0 & i=1, \cdots, m, \\
s_{r}^{+} \geq 0 & r=1, \cdots, s .
\end{array}
$$

where $\theta_{o}, \lambda_{j}, \rho_{w j}, s_{i}^{-}$, and $s_{r}^{+}$are the dual variables. $\theta_{o}$ is radial input shrinkage factor (eventually to become efficiency measure) and $\lambda=\left\{\lambda_{j}\right\}$ is vector of DMU loadings, determining "best practice" for 
the DMU being evaluated. $c_{j o}^{U}$ stands for the best possible relative efficiency achieved by $\mathrm{DMU}_{o}$ when all the DMUs are in the state of best production activity, while $c_{j o}^{L}$ stands for the lower bound of the best possible relative efficiency of $\mathrm{DMU}_{o}$. They constitute a possible best relative efficiency interval $\left[c_{j o}^{L}, c_{j o}^{U}\right]$. The variable $s_{r}^{+}$is shortfall amount of output $r$ and $s_{i}^{-}$is excess amount of input $i$. From the duality theory in linear programming, for an inefficient $\mathrm{DMU}_{o}, \lambda_{j}^{*}>0$ in the optimal dual solution implies that $\mathrm{DMU}_{j}$ is a unit of the peer group. A peer group of an inefficient $\mathrm{DMU}_{o}$ is defined as the set of DMUs that reach the efficiency score of 1 using the same set of weights that result in the efficiency score of $\mathrm{DMU}_{o}$. It is the existence of this collection of DMUs that forces the $\mathrm{DMU}_{o}$ to be inefficient.

Now, suppose that the input variables may be partitioned into subsets of discretionary (D) and nondiscretionary $(\mathrm{N})$ variables. Thus,

$$
I=\{1,2, \ldots, m\}=I_{D} \cup I_{N}, \quad I_{D} \cap I_{N}=\Phi
$$

The pair of AR-NF-IDEA models is then finally given by 
$\operatorname{Minc}_{j o}^{U}=\theta_{O}-\sum_{j=1}^{n} \sum_{w=1}^{t} k_{w} \rho_{w j}-\varepsilon \sum_{i \in D}^{m} s_{i}^{-}-\varepsilon \sum_{r=1}^{s} s_{r}^{+}$,

s.t.

$$
\begin{aligned}
& \sum_{j=1}^{n} y_{r j}^{U} \lambda_{j}-\sum_{j=1}^{n} \sum_{w=1}^{t} b_{r w} y_{r j}^{U} \rho_{w j}-s_{r}^{+}=y_{r j o}^{U} \quad r=1, \cdots, s, \\
& x_{i j o}^{L} \theta_{O}-\sum_{j=1}^{n} x_{i j}^{L} \lambda_{j}-\sum_{j=1}^{n} \sum_{w=1}^{t} a_{i w} x_{i j}^{L} \rho_{w j}-s_{i}^{-}=0 \\
& x_{i j o}^{L}-\sum_{j=1}^{n} x_{i j}^{L} \lambda_{j}-\sum_{j=1}^{n} \sum_{w=1}^{t} a_{i w} x_{i j}^{L} \rho_{w j}-s_{i}^{-}=0 \quad i \in D, \\
& \theta_{O} \quad \text { free, } \\
& \rho_{w j} \geq 0 \quad \forall w, j, \\
& \lambda_{j} \geq 0 \quad j=1, \cdots, n, \\
& s_{i}^{-} \geq 0 \quad i \in D, \\
& s_{i}^{-}=0 \quad i \in N, \\
& s_{r}^{+} \geq 0 \quad r=1, \cdots, s .
\end{aligned}
$$

$\operatorname{Minc}_{j o}^{L}=\theta_{O}-\sum_{j=1}^{n} \sum_{w=1}^{t} k_{w} \rho_{w j}-\varepsilon \sum_{i \in D}^{m} s_{i}^{-}-\varepsilon \sum_{r=1}^{S} s_{r}^{+}$,

s.t.

$$
\begin{aligned}
& \sum_{j=1}^{n} y_{r j}^{U} \lambda_{j}-\sum_{j=1}^{n} \sum_{w=1}^{t} b_{r w} y_{r j}^{U} \rho_{w j}-s_{r}^{+}=y_{r j o}^{L} \quad r=1, \cdots, s, \\
& x_{i j o}^{U} \theta_{O}-\sum_{j=1}^{n} x_{i j}^{L} \lambda_{j}-\sum_{j=1}^{n} \sum_{w=1}^{t} a_{i w} x_{i j}^{L} \rho_{w j}-s_{i}^{-}=0 \quad i \in D, \\
& x_{i j o}^{U}-\sum_{j=1}^{n} x_{i j}^{L} \lambda_{j}-\sum_{j=1}^{n} \sum_{w=1}^{t} a_{i w} x_{i j}^{L} \rho_{w j}-s_{i}^{-}=0 \quad i \in N, \\
& \theta_{0} \quad \text { free, } \\
& \rho_{w j} \geq 0 \quad \forall w, j, \\
& \lambda_{j} \geq 0 \quad j=1, \cdots, n, \\
& s_{i}^{-} \geq 0 \quad i \in D \text {, } \\
& s_{i}^{-}=0 \quad i \in N \text {, } \\
& s_{r}^{+} \geq 0 \quad r=1, \cdots, s .
\end{aligned}
$$


It is to be noted that the $\theta_{o}$ to be minimized appears only in the constraints for which $i \in D$, whereas the constraints for which $i \in N$ operate only indirectly (as they should) because the input levels $x_{i j o}^{U}$ are not subject to managerial control. Therefore this is recognized by entering all $x_{i j o}^{U}, \quad i \in N$ at their fixed (observed) value. Note that the slacks $s_{i}^{-}, i \in N$ are omitted from the objective function. Hence these nondiscretionary inputs do not enter directly into the efficiency measures being optimized in (16) and (17). They can, nevertheless, affect the efficiency evaluations by virtue of their presence in the constraints. For models (16) and (17), it is not relevant to minimize the proportional decrease in the entire input vector. Such minimization should be determined only with respect to the subvector that is composed of discretionary inputs.

In order to judge whether a DMU is DEA efficient or not, the following definition is given.

Definition 2. A DMU, $\mathrm{DMU}_{o}$, is said to be DEA efficient if its best possible upper bound efficiency $c_{j o}^{U^{*}}=1$; otherwise, it is said to be DEA inefficient if $c_{j o}^{U^{*}}<1$.

Therefore, one unified model that deals with all aspects of the weights restrictions, imprecise data and nondiscretionary factors in a direct manner have been introduced ${ }^{1}$.

Now, the method of transforming ordinal preference information into interval data is discussed, so that the pair of AR-NF-IDEA models presented in this paper can still work properly even in these situations.

Suppose some input and/or output data for DMUs are given in the form of ordinal preference information. Usually, there may exist three types of ordinal preference information: (1) strong ordinal preference information such as $y_{r j}>y_{r k}$ or $x_{i j}>x_{i k}$, which can be further expressed as $y_{r j} \geq \chi_{r} y_{r k}$ and $x_{i j} \geq \eta_{i} x_{i k}$, where $\chi_{r}>1$ and $\eta_{i}>1$ are the parameters on the degree of preference intensity provided by decision maker; (2) weak ordinal preference information such as $y_{r p} \geq y_{r q}$ or $x_{i p} \geq x_{i q}$; (3) indifference relationship such as $y_{r l}=y_{r t}$ or $x_{i l}=x_{i t}$. Since DEA model has the property of unit-invariance, the use of scale transformation to ordinal preference information does not change the original ordinal relationships and has no effect on the efficiencies of DMUs. Therefore, it is possible to conduct a scale transformation to every ordinal input and output index so that its best ordinal datum is less than or equal to unity and then give an interval estimate for each ordinal datum.

1 Note that the Models (10) and (11) could be solved by one of the optimization techniques (e.g., Lingo software). Since Models (10) and (11) are linear, so the computations will be done quickly and global optimum solution will be found. Meanwhile, reducing number of inputs and outputs will ensure better discrimination between the DMUs being analyzed. There is a rule of thumb wherein the number of DMUs should be at least three times the total number of inputs and outputs. Therefore, reducing the dimensionality of the problem will result in less DMUs being efficient and thus more knowledge gained. 
Now, consider the transformation of ordinal preference information about the output $y_{r j}(j=1, \ldots, n)$ for example. The ordinal preference information about input and other output data can be converted in the same way.

For weak ordinal preference information $y_{r 1} \geq y_{r 2} \geq \cdots \geq y_{r n}$, we have the following ordinal relationships after scale transformation:

$$
1 \geq \hat{y}_{r 1} \geq \hat{y}_{r 2} \geq \cdots \geq \hat{y}_{r n} \geq \sigma_{r}
$$

where $\sigma_{r}$ is a small positive number reflecting the ratio of the possible minimum of $\left\{y_{r j} \mid j=1, \ldots, n\right\}$ to its possible maximum. It can be approximately estimated by the decision maker. It is referred as the ratio parameter for convenience. The resultant permissible interval for each $\hat{y}_{r j}$ is given by

$$
\hat{y}_{r j} \in\left[\sigma_{r}, 1\right], \quad j=1, \cdots, n \text {. }
$$

For strong ordinal preference information $y_{r 1}>y_{r 2}>\cdots>y_{r n}$, there is the following ordinal relationships after scale transformation:

$$
1 \geq \hat{y}_{r 1}, \quad \hat{y}_{r j} \geq \chi_{r} \hat{y}_{r, j+1} \quad(j=1, \cdots, n-1) \quad \text { and } \quad \hat{y}_{r n} \geq \sigma_{r} \text {, }
$$

where $\chi_{r}$ is a preference intensity parameter satisfying $\chi_{r}>1$ provided by the decision maker and $\sigma_{r}$ is the ratio parameter also provided by the decision maker. The resultant permissible interval for each $\hat{y}_{r j}$ can be derived as follows:

$$
\hat{y}_{r j} \in\left[\sigma_{r} \chi_{r}^{n-j}, \chi_{r}^{1-j}\right] \quad j=1, \cdots, n \text { with } \quad \sigma_{r} \leq \chi_{r}^{1-n} .
$$

Finally, for indifference relationship, the permissible intervals are the same as those obtained for weak ordinal preference information.

Through the scale transformation above and the estimation of permissible intervals, all the ordinal preference information is converted into interval data and can thus be incorporated into the pair of ARNF-IDEA models.

The following subsection lists the nomenclature used to formulate the problem under consideration.

\subsection{Nomenclature}

$j=1, \ldots, n \quad$ collection of technologies (DMUs)

$j_{o}, D M U_{o}=$ the DMU under evaluation

$r=1, \ldots, s \quad$ the set of outputs

$i=1, \ldots, m \quad$ the set of inputs

$y_{r j}=$ the $r$ th output of $j$ th DMU

$x_{i j}=$ the $i$ th input of $j$ th DMU

$y_{r k}=$ the $r$ th output of $k$ th DMU related to strong ordinal preference information

$x_{i k}=$ the $i$ th input of $k$ th DMU related to strong ordinal preference information

$y_{r p}, y_{r q}=$ the $r$ th output of the DMU related to weak ordinal preference information

$x_{i p}, x_{i q}=$ the $i$ th input of the DMU related to weak ordinal preference information 
$y_{r l}, y_{r t}=$ indifference relationship among outputs

$x_{i l}, x_{i t}=$ indifference relationship among inputs

$y_{r j}^{L}, x_{i j}^{L}=$ lower bound

$y_{r j}^{U}, x_{i j}^{U}=$ upper bound

$\theta_{j o}^{U}=$ the best possible relative efficiency achieved by $\mathrm{DMU}_{o}$

$\theta_{j o}^{L}=$ the lower bound of the best possible relative efficiency of $\mathrm{DMU}_{o}$

$\theta_{o}=$ radial input shrinkage factor (eventually to become efficiency measure of $\mathrm{DMU}_{o}$ )

$\chi_{r}, \eta_{i}=$ the parameters on the degree of preference intensity provided by decision maker

$\sigma_{r}=$ small positive number reflecting the ratio of the possible minimum of $\left\{y_{r j} \mid j=1, \ldots, n\right\}$ to its possible maximum

$\hat{y}_{r j}=$ the $r$ th output of $j$ th DMU after scale transformation

$u_{r}=$ weight of the $r$ th output

$v_{i}=$ weight of the ith input

$\lambda_{\mathrm{j}}=$ vector of DMU loadings, determining "best practice" for the DMU being evaluated

$\delta_{i}=$ the lower bound of the ith input weight

$\tau_{i}=$ the upper bound of the ith input weight

$\rho_{r}=$ the lower bound of the $r$ th output weight

$\eta_{r}=$ the upper bound of the $r$ th output weight

$\alpha_{i}=$ the lower bound of relative weight restrictions of the $i$ th input

$\beta_{i}=$ the upper bound of relative weight restrictions of the ith input

$\theta_{r}=$ the lower bound of relative weight restrictions of the $r$ th output

$\zeta_{r}=$ the upper bound of relative weight restrictions of the $r$ th output

$\gamma_{i}=$ input-output weight restriction of assurance regions of type II

$a_{r}=$ the lower bound of $r$ th virtual output

$b_{r}=$ the upper bound of $r$ th virtual output

$c_{i}=$ the lower bound of $i$ th virtual input

$d_{i}=$ the upper bound of $i$ th virtual input

$w=1, \ldots, t \quad$ set of virtual weights restrictions

$a_{i w}=$ the weight of $i$ th input in $w$ th virtual weight restriction

$b_{r w}=$ the weight of $r$ th output in wth virtual weight restriction

$i^{\prime}=$ the virtual input

$r^{\prime}=$ the virtual output

$\rho_{w j}=$ dual variable concerned to virtual weights restrictions

$k_{w}=$ set of $w$ th virtual weights restrictions

$s_{i}^{-}=$excess amount of input $i$

$s_{r}^{+}=$shortfall amount of output $r$

$c_{j o}^{U}=$ the best possible relative efficiency achieved by $\mathrm{DMU}_{o}$ 
$c_{j o}^{L}=$ the lower bound of the best possible relative efficiency of $\mathrm{DMU}_{o}$.

$D=$ subsets of discretionary variables

$N=$ subsets of nondiscretionary variables

$I_{D}=$ the subsets of discretionary inputs

$I_{N}=$ the subsets of nondiscretionary inputs

In the next section, a numerical example is presented.

\section{Numerical example}

In this section, the proposed methodology that may be applied to a wide range of technology selection problems is used for robot selection, and is illustrated through a previously reported industrial robot selection problem. Farzipoor Saen [7] applied DEA to identify the optimal robot. In particular, this example is used to show how ordinal data, bounded data, and weight restriction as well as nondiscretionary factors, can be combined into the one unified approach provided by AR-NF-IDEA. The robot selection problem addressed in Farzipoor Saen [7] involves the evaluation of relative efficiency of 27 robots with respect to attributes including "load capacity", and "velocity", which are considered in some sense as outputs, and "cost" and "vendor reputation", which are considered in some sense as inputs. "Vendor reputation" is included as a qualitative input while "load capacity" will serve as the bounded data output. "Vendor reputation" is an intangible factor which is not usually explicitly included in evaluation model for technology. This qualitative variable is measured on an ordinal scale so that, for instance, technology 23 is given the highest rank, and technology 17, the lowest. Note that these measures are not exhaustive by any means, but frequently used in robot's performance evaluation. In an application of this methodology, DMs must carefully identify appropriate inputs and outputs to be used in the decision making process. Assume that "cost" is a cardinal input and because of limitation of number of suppliers of this kind of technology (oligopolistic environment), this factor is considered as nondiscretionary factor. Table 1 depicts the robot attributes. 
Table 1. Related attributes for 27 robots

\begin{tabular}{|c|c|c|c|c|}
\hline \multirow[b]{2}{*}{$\begin{array}{c}\text { Robot } \\
\text { No. } \\
\text { (DMU) }\end{array}$} & \multicolumn{2}{|r|}{ Inputs } & \multicolumn{2}{|c|}{ Outputs } \\
\hline & $\begin{array}{c}\text { Cost } \\
(10000 \$) \\
x_{1 j}\end{array}$ & $\begin{array}{c}\text { Vendor Reputation } \\
x_{2 j}\end{array}$ & $\begin{array}{c}\text { Load } \\
\text { Capacity } \\
(\mathrm{kg}) \\
y_{1 j}\end{array}$ & $\begin{array}{c}\text { Velocity } \\
(\mathrm{m} / \mathrm{s}) \\
y_{2 j}\end{array}$ \\
\hline 1 & 7.2 & 15 & {$[50,65]$} & 1.35 \\
\hline 2 & 4.8 & 7 & {$[60,70]$} & 1.1 \\
\hline 3 & 5 & 23 & {$[40,50]$} & 1.27 \\
\hline 4 & 7.2 & 16 & {$[1,3]$} & .66 \\
\hline 5 & 9.6 & 24 & {$[45,55]$} & .05 \\
\hline 6 & 1.07 & 3 & {$[1,2]$} & .3 \\
\hline 7 & 1.76 & 8 & {$[4,5]$} & 1 \\
\hline 8 & 3.2 & 17 & {$[10,20]$} & 1 \\
\hline 9 & 6.72 & 9 & {$[9,12]$} & 1.1 \\
\hline 10 & 2.4 & 2 & {$[5,8]$} & 1 \\
\hline 11 & 2.88 & 18 & {$[25,35]$} & .9 \\
\hline 12 & 6.9 & 10 & {$[10,15]$} & .15 \\
\hline 13 & 3.2 & 25 & {$[8,12]$} & 1.2 \\
\hline 14 & 4 & 19 & {$[20,35]$} & 1.2 \\
\hline 15 & 3.68 & 11 & {$[40,55]$} & 1 \\
\hline 16 & 6.88 & 20 & {$[75,85]$} & 1 \\
\hline 17 & 8 & 1 & {$[10,18]$} & 2 \\
\hline 18 & 6.3 & 21 & {$[9,15]$} & 1 \\
\hline 19 & .94 & 12 & {$[10,13]$} & .3 \\
\hline 20 & .16 & 5 & {$[1,4]$} & .8 \\
\hline 21 & 2.81 & 26 & {$[25,30]$} & 1.7 \\
\hline 22 & 3.8 & 13 & {$[0.8,1.2]$} & 1 \\
\hline 23 & 1.25 & 27 & {$[2,4]$} & .5 \\
\hline 24 & 1.37 & 14 & {$[1,5]$} & .5 \\
\hline 25 & 3.63 & 4 & {$[8,12]$} & 1 \\
\hline 26 & 5.3 & 22 & {$[65,80]$} & 1.25 \\
\hline 27 & 4 & 6 & {$[190,220]$} & .75 \\
\hline
\end{tabular}


Suppose the preference intensity parameter and the ratio parameter about the strong ordinal preference information are given (or estimated) as $\eta_{2}=1.12$ and $\sigma_{2}=0.01$, respectively. Using the transformation technique described in previous section, an interval estimate for "vendor reputation" of each technology can be derived, which is shown in the Table 2.

Table 2. Interval estimate for the 27 robots after the transformation of ordinal preference information

\begin{tabular}{|c|c|}
\hline $\begin{array}{c}\text { Robot } \\
\text { No. } \\
\text { (DMU) }\end{array}$ & Vendor Reputation \\
\hline 1 & {$[.0488711, .2566751]$} \\
\hline 2 & {$[.0197382, .1036668]$} \\
\hline 3 & {$[.1210031, .635518]$} \\
\hline 4 & {$[.0547357, .2874761]$} \\
\hline 5 & {$[.1355235, .7117802]$} \\
\hline 6 & {$[.012544, .0658821]$} \\
\hline 7 & {$[.0221068, .1161068]$} \\
\hline 8 & {$[.0613039, .3219732]$} \\
\hline 9 & {$[.0247596, .1300396]$} \\
\hline 10 & {$[.0112, .0588233]$} \\
\hline 11 & {$[.0686604, .36061]$} \\
\hline 12 & {$[.0277308, .1456443]$} \\
\hline 13 & {$[.1517863, .7971939]$} \\
\hline 14 & {$[.0768997, .4038832]$} \\
\hline 15 & {$[.0310585, .1631217]$} \\
\hline 16 & {$[.0861276, .4523492]$} \\
\hline 17 & {$[.01, .0525208]$} \\
\hline 18 & {$[.0964629, .5066311]$} \\
\hline 19 & {$[.0347855, .1826963]$} \\
\hline 20 & {$[.0157352, .0826425]$} \\
\hline 21 & {$[.1700006, .8928571]$} \\
\hline 22 & {$[.0389598, .2046198]$} \\
\hline 23 & {$[.1904007,1]$} \\
\hline 24 & {$[.0436349, .2291742]$} \\
\hline 25 & {$[.0140493, .073788]$} \\
\hline 26 & {$[.1080385, .5674269]$} \\
\hline 27 & {$[.0176234, .0925596]$} \\
\hline & \\
\hline
\end{tabular}

Therefore, all the input and output data are now transformed into interval numbers and can be evaluated using the pair of AR-NF-IDEA models.

According to the decision of DM, the importance of cost must be greater than vendor reputation. Assume that cost is twice as important as vendor reputation (virtual assurance regions of type I). 
Applying Models (16) and (17), the efficiency scores of robots (DMUs) and peer groups of robots have been presented in Table 3. The positive non-Archimedean infinitesimal, $\varepsilon$ has been set to 0.0001 .

Table 3. The efficiency interval and peer group for the 27 robots

\begin{tabular}{|c|c|c|}
\hline $\begin{array}{c}\text { Robot } \\
\text { No. } \\
\text { (DMU) }\end{array}$ & Efficiency Interval & Peer group \\
\hline 1 & {$[.03984, .22832]$} & 17,27 \\
\hline 2 & {$[.08549, .48048]$} & 17,27 \\
\hline 3 & {$[.01424, .08168]$} & $10,17,27$ \\
\hline 4 & {$[.0209, .11206]$} & 17 \\
\hline 5 & {$[.01095, .07215]$} & 27 \\
\hline 6 & {$[.03023, .15953]$} & 17,20 \\
\hline 7 & {$[.11727, .61716]$} & 17,20 \\
\hline 8 & {$[.02555, .14779]$} & $10,17,27$ \\
\hline 9 & {$[.05336, .28597]$} & 17,27 \\
\hline 10 & {$[.18977,1]$} & $\mathrm{N} / \mathrm{A}$ \\
\hline 11 & {$[.02423, .13943]$} & $10,17,27$ \\
\hline 12 & [.03587, .19962] & 17,27 \\
\hline 13 & {$[.01512, .08159]$} & $10,17,27$ \\
\hline 14 & {$[.02454, .14515]$} & $10,17,27$ \\
\hline 15 & {$[.05422, .32508]$} & $10,17,27$ \\
\hline 16 & {$[.0241, .13377]$} & 17,27 \\
\hline 17 & {$[.1896,1]$} & N/A \\
\hline 18 & {$[.01271, .0706]$} & 17,27 \\
\hline 19 & {$[.01713, .09719]$} & $10,17,27$ \\
\hline 20 & {$[.1901,1]$} & N/A \\
\hline 21 & {$[.02729, .1497]$} & $10,20,27$ \\
\hline 22 & {$[.02741, .1474]$} & 17,20 \\
\hline 23 & {$[.00418, .02839]$} & $10,17,20$ \\
\hline 24 & {$[.02124, .11495]$} & $10,17,27$ \\
\hline 25 & {$[.08706, .47714]$} & $10,17,27$ \\
\hline 26 & {$[.01723, .10006]$} & $10,17,27$ \\
\hline 27 & {$[.170265,1]$} & N/A \\
\hline
\end{tabular}

Based on the definition 2, robots 10, 17, 20, and 27 have the possibility to be DEA efficient. If they are able to use the minimum inputs to produce the maximum outputs, they are DEA efficient (efficient in scale); otherwise, they are not DEA efficient. The remaining 23 robots with relative efficiency scores of 
less than 1 are considered to be inefficient. Therefore, DM can choose one or more of these efficient robots. Also, the last column of Table 3 provides peer groups for inefficient robots.

Note that, each DEA model seeks to determine which of the $n$ DMUs define an envelopment surface that represents best practice, referred to as the empirical production function or the efficient frontier. DMUs that lie on the surface are deemed efficient in DEA while those DMUs that do not, are termed inefficient. DEA provides a comprehensive analysis of relative efficiencies for multiple input-multiple output situations by evaluating each DMU and measuring its performance relative to an envelopment surface composed of other DMUs. Those DMUs are the peer group for the inefficient DMUs known as the efficient reference set. As the inefficient DMUs are projected onto the envelopment surface, the efficient DMUs closest to the projection and whose linear combination comprises this virtual DMU form the peer group for that particular DMU. The targets defined by the efficient projections give an indication of how this DMU can improve to be efficient. The peer groups serve as a benchmark to use in seeking improvements for inefficient robots. Inefficient robot suppliers can use these results from a marketing perspective.

\section{Concluding remarks}

DEA has previously been used for the selection of technologies. Such a linear programming technique seems to be able to support management in the critical and delicate task of selecting the most efficient technology among a set of competing multi-attribute technologies. This paper has introduced a new pair of AR-NF-IDEA models and employed it for technology selection. Also, this paper proposed a systematic analysis to provide peer groups for inefficient technology suppliers. The proposed methodology can be applied from both a buyer's and technology supplier's perspective. The buyer can use it as a tool in selecting the "best" technology. The technology supplier can use these results from a marketing perspective. A specific technology supplier who achieves a high mean score, when compared to the other technology suppliers, can use these results for promoting their technology. On the other hand, if a particular technology is poorly performing, then the technology supplier can use the analysis for benchmarking purposes. This result may mean that the technology supplier must provide better performance levels at the same cost. Ultimately, the ability of a technology supplier to scan the technological environment may lead to the establishing of sound action guidelines aimed at improving a technology supplier’s long-term competitive position.

The problem considered in this study is at initial stage of investigation and much further researches can be done based on the results of this paper. Some of them are as follows:

Similar research can be repeated for technology ranking in the presence of imprecise data, weight restrictions, and nondiscretionary factors.

In this study, the proposed model has been applied to a problem related to robot selection. However, the same models could be applied, with minor modifications, to other problems related to selection of 
flexible manufacturing systems, computer integrated manufacturing systems, computer numerical control systems and many other technology selection decision cases.

\section{Acknowledgements}

The author wishes to thank two anonymous reviewers for their valuable suggestions and comments.

\section{References}

[1] Rai R., Kameshwaran S., Tiwari M. K. (2002) Machine-Tool Selection and Operation Allocation in FMS: Solving a Fuzzy Goal-Programming Model Using a Genetic Algorithm, International Journal of Production Research, Vol. 40, No. 3, pp. 641-665.

[2] Chan F. T. S., Swarnkar R., Tiwari M. K. (2005) Fuzzy Goal-Programming Model with an Artificial Immune System (AIS) Approach for a Machine Tool Selection and Operation Allocation Problem in a Flexible Manufacturing System, International Journal of Production Research, Vol. 43, No. 19, pp. 41474163.

[3] Jaganathan S., Erinjeri J. J., Ker J. (2007) Fuzzy Analytic Hierarchy Process Based Group Decision Support System to Select and Evaluate New Manufacturing Technologies, International Journal of Advanced Manufacturing Technology, Vol. 32, No. 11-12, pp. 1253-1262.

[4] Khouja M. (1995) The Use of Data Envelopment Analysis for Technology Selection, Computers \& Industrial Engineering, Vol. 28, No. 1, pp. 123-132.

[5] Baker R. C., Talluri S. (1997) A Closer Look at the Use of Data Envelopment Analysis for Technology Selection, Computers \& Industrial Engineering, Vol. 32, No. 1, pp. 101-108.

[6] Ramanathan R. (2001) Comparative Risk Assessment of Energy Supply Technologies: A Data Envelopment Analysis Approach, Energy, Vol. 26, No. 2, pp. 197-203.

[7] Farzipoor Saen R. (2006) A decision model for technology selection in the existence of both cardinal and ordinal data, Applied Mathematics and Computation, Vol. 181, No. 2, pp. 1600-1608.

[8] Talluri S., Whiteside M. M., Seipel S. J. (2000) A Nonparametric Stochastic Procedure for FMS Evaluation, European Journal of Operational Research, Vol. 124, No. 3, pp. 529-538.

[9] Seiford L. M., Zhu J. (2003) Context-Dependent Data Envelopment Analysis-Measuring Attractiveness and Progress, Omega, Vol. 31, No. 5, pp. 397-408.

[10] Karsak E. E., Ahiska S. S. (2005) Practical Common Weight Multi-Criteria Decision-Making Approach with an Improved Discriminating Power for Technology Selection, International Journal of Production Research, Vol. 43, No. 8, pp. 1537-1554. 
[11] Sarkis J., Talluri S. (1999) A Decision Model for Evaluation of Flexible Manufacturing Systems in the Presence of both Cardinal and Ordinal Factors, International Journal of Production Research, Vol. 37, No. 13, pp. 2927-2938.

[12] Cook W. D., Kress M., Seiford L. M. (1996) Data Envelopment Analysis in the Presence of both Quantitative and Qualitative factors, Journal of Operational Research Society, Vol. 47, No. 7, pp. 945953.

[13] Talluri S., Yoon K.P. (2000) A cone-ratio DEA Approach for AMT Justification, International Journal of Production Economics, Vol. 66, No. 2, pp. 119-129.

[14] Shang J., Sueyoshi T. (1995) A Unified Framework for the selection of Flexible Manufacturing System, European Journal of Operational Research, Vol. 85, No. 2, pp. 297-315.

[15] Braglia M., Petroni A. (1999) Evaluating and Selecting Investments in Industrial Robots, International Journal of Production Research, Vol. 37, No. 18, pp. 4157-4178.

[16] Farzipoor Saen R. (2006) A decision model for selecting technology suppliers in the presence of nondiscretionary factors, Applied Mathematics and Computation, Vol. 181, No. 2, pp. 1609-1615.

[17] Farzipoor Saen R. (2006) An algorithm for ranking technology suppliers in the presence of nondiscretionary factors, Applied Mathematics and Computation, Vol. 181, No. 2, pp. 1616-1623.

[18] Charnes A., Cooper W. W., Rhodes E. (1978) Measuring the Efficiency of Decision Making Units, European Journal of Operational Research, Vol. 2, No. 6, pp. 429-444.

[19] Banker R. D., Charnes A., Cooper W. W. (1984) Some Methods for Estimating Technical and Scale Inefficiencies in Data Envelopment Analysis, Management Science, Vol. 30, No. 9, pp. 1078-1092.

[20] Charnes A., Cooper W. W., Wei Q. L., Huang Z. M. (1989) Cone-ratio Data Envelopment Analysis and Multi-objective Programming, International Journal of Systems Sciences, Vol. 20, No. 7, pp. 10991118.

[21] Thompson R. G., Langemeier L. N., Lee C. T., Lee E., Thrall R. M. (1990) The Role of Multiplier Bounds in Efficiency Analysis with Application to Kansas Farming, Journal of Econometrics, Vol. 46, No. 1/2, pp. 93-108.

[22] Wong Y. H. B., Beasley, J. E. (1990) Restricting Weight Flexibility in Data Envelopment Analysis, Journal of the Operational Research Society, Vol. 41, No. 9, pp. 829-835.

[23] Sarrico C. S., Dyson R. G. (2004) Restricting Virtual Weights in Data Envelopment Analysis, European Journal of Operational Research, Vol. 159, No. 1, pp. 17-34.

[24] Cooper W. W., Park K. S., Yu G. (1999) IDEA and AR-IDEA: Models for Dealing with Imprecise Data in DEA, Management Science, Vol. 45, No. 4, pp. 597-607.

[25] Cooper W. W., Park K. S., Yu G. (2001) An Illustrative Application of IDEA (Imprecise Data Envelopment Analysis) to a Korean Mobile Telecommunication Company, Operations Research, Vol. 49, No. 6, pp. 807-820. 
[26] Cooper W. W., Park K. S., Yu G. (2001) IDEA (Imprecise Data Envelopment Analysis) with CMDs (Column Maximum Decision Making Units), Journal of the Operational Research Society, Vol. 52, No. 2, pp. 176-181.

[27] Wang Y. M., Greatbanks R. and Yang J. B. (2005) Interval Efficiency Assessment Using Data Envelopment Analysis, Fuzzy Sets and Systems, Vol. 153, No. 3, pp. 347-370.

[28] Despotis D. K., Smirlis Y. G. (2002) Data Envelopment Analysis with Imprecise Data, European Journal of Operational Research, Vol. 140, No. 1, pp. 24-36.

[29] Zhu J. (2003) Imprecise Data Envelopment Analysis (IDEA): A Review and Improvement with an Application, European Journal of Operational Research, Vol. 144, No. 3, pp. 513-529. 